\title{
Costos derivados de la atención a pacientes con enfermedad crónica no transmisible ${ }^{1}$
}

\author{
Costs derivative of the attention of patients with non transferable \\ chronic disease
}

\begin{abstract}
María Stella Campos-de Aldana², Myriam Durán-Parra ${ }^{3}$, Sonia Solano-Aguilar ${ }^{4}$ Delia Moya-Plata ${ }^{5}$, Ligia Betty ArboledaSalazar $^{6}$, Erika Yurley Duran-Niño ${ }^{7}$, Julieth Dayana Mendoza-Matajira ${ }^{8}$
\end{abstract}

\begin{abstract}
Forma de citar: M. Campos, M. Durán, S. Solano, et al, "Costos derivados de la atención a pacientes con enfermedad crónica no transmisible”. Respuestas. vol. 19, no. 2, pp. 51-58, 2014.
\end{abstract}

Recibido:

Diciembre 2 de 2013

Aceptado:

Marzo 13 de 2014

${ }^{1}$ Articulo de Revisión. Se desarrolló en el marco del proyecto de investigación "Carga de la enfermedad crónica no transmisible en Colombia", trabajo financiado por Colciencias, Universidad Nacional de Colombia

(UNAL), Universidad de Santander

(UDES) y Universidad de Ciencias

Aplicadas y Ambientales (U.D.C.A).

${ }^{2}$ Magister en Salud Familiar

Universidad de Santander UDES Bucaramanga-Colombia

${ }^{3}$ Magíster en Enfermería con Énfasis en Gerencia de Servicios de Salud enfermería@udes.edu.co Universidad de Santander UDES Bucaramanga-Colombia

${ }^{4}$ Especialista en Administración de Servicios

Universidad de Santander UDES Bucaramanga-Colombia

${ }^{5}$ Magíster en Salud Familiar Universidad de Santander UDES Bucaramanga- Colombia

${ }^{6}$ Especialista en Docencia Universitaria

Universidad de Santander UDES Bucaramanga-Colombia

${ }^{7}$ Enfermera

Universidad de Santander UDES Bucaramanga- Colombia

${ }^{8}$ Enfermera

Universidad de Santander UDES Bucaramanga- Colombia

\section{Resumen}

Antecedentes: Las enfermedades crónicas no transmisibles, son la principal causa de morbimortalidad a nivel mundial. Comportamiento similar se presenta en Colombia al igual que en Santander. Las patologías cardiovasculares en el 2010 cobraron la vida de 1527 mujeres y 1634 hombres; seguido de las neoplasias, en especial las de localización de tumores malignos que causaron la muerte a 727 mujeres y 755 hombres, estos resultados se ven reflejados en los altos costos requeridos para la atención hospitalaria, ambulatoria y domiciliaria por lo que requiere dedicación, educación y cuidado individualizado. Métodos: Se realizó una búsqueda de artículos teniendo en cuenta tiempo de publicación y las palabras claves; en las bases de datos: MEDLINE, IME, LILACS, Centro Cochrane Iberoamericano, EMBASE, CINAHL, BDIE, en el primer semestre de 2013. Identificando 55 estudios potenciales, de los cuales se seleccionaron seis artículos del comportamiento de los costos de la atención a pacientes con enfermedades crónicas no transmisibles; cabe incluir que se obtuvo información directa por parte de algunas instituciones de Bucaramanga, la cual demuestra el comportamiento de los costos frente al cuidado de enfermería y a nivel interno de cada entidad no solo del sistema de salud sino de los pacientes y cuidadores familiares. Resultados: la enfermedad crónica genera la mayor parte de los costos al Sistema de Salud, situación a tener en cuenta; así como el cuidado efectivo para la atención de pacientes que ya han sido afectados. Conclusiones: el Sistema de Salud debe implementar estrategias preventivas para la aparición de las Enfermedades Crónicas No Trasmisibles (ECNT) y las complicaciones derivadas de ellas, que contemple un cuidado efectivo para la atención de los individuos.

Palabras claves: Costos, Cuidado, Enfermedad Crónica no transmisible. 


\begin{abstract}
Background: Chronic noncommunicable diseases are the leading cause of morbidity and mortality worldwide. Similar behavior occurs in Colombia as in Santander. Cardiovascular diseases in 2010 claimed the lives of 1527 women and 1634 men; followed by neoplasms, especially the localization of malignant tumors that killed 727 women and 755 men, these results are reflected in the high cost required for inpatient, outpatient and home care so requires dedication, education and individualized care. Methods: A search of articles were given at time of publication and keyword; in databases: MEDLINE, IME, LILACS, Iberoamerican Cochrane Centre, EMBASE, CINAHL, BDIE in the first half of 2013. By identifying 55 potential studies, of which six articles of behavior of costs of care were selected patients with chronic non- communicable diseases; it should include direct information was obtained by some institutions Bucaramanga, which shows the behavior of costs compared to nursing care and internally each institution not only the health system but patients and family caregivers. Results: Chronic illness generates most of the costs the health system, a situation to consider; and effective care for the care of patients who have already been affected. Conclusions: Health System must implement preventive strategies for the appearance of Chronic Non Communicable (ECNT) and complications arising from them, by providing effective care for the care of individuals.
\end{abstract}

Keywords: Costs, Care, Chronic Non-Communicable Disease.

\section{Introducción}

Al igual que en el resto del mundo, la principal causa de muerte en Santander es la enfermedad cardiovascular, cobrando en el 2010 la vida de 1527 mujeres y 1634 hombres. Así mismo, se identifica un aumento en las neoplasias, en especial las de localización de tumores malignos que causaron la muerte a 727 mujeres y 755 hombres. En Bucaramanga, capital de Santander (Colombia), según los Indicadores Básicos de Salud 2010 se registró una tasa de 183.6 x 100.000 habitantes por muerte a causa de enfermedades cardiovasculares, esta tasa se disgrega de la siguiente manera: por enfermedades del sistema circulatorio, 159.7, mortalidad por enfermedad isquémica del corazón en personas de 45 y más años de edad, 297.5, mortalidad por enfermedades cerebrovasculares en personas mayores de 45 años de edad, 145.4, mortalidad por diabetes mellitus en personas de 45 y más años es
86.5, todas estas tasas calculadas por 100.000 habitantes.

Según Jova y otros. (2011) las enfermedades crónicas son uno de los mayores retos que enfrentan los sistemas de salud debido a varios factores: gran número de casos afectados, creciente contribución a la mortalidad general, conformación en la causa más frecuente de incapacidad prematura y la complejidad y costo elevado de su tratamiento situación que en Bucaramanga se ve reflejada en sus estadísticas de mortalidad.

\section{Materiales y métodos}

Revisión Sistemática de artículos de diferentes bases de datos MEDLINE, IME, LILACS, Centro Cochrane Iberoamericano, EMBASE, CUIDEN, CINAHL, BDIE, durante el primer semestre de 2013, publicados entre los años 
María Stella Campos-de Aldana, Myriam Durán-Parra, Sonia Solano-Aguilar, Delia Moya-Plata, Ligia Betty Arboleda-Salazar, Erika Yurley Duran-Niño, Julieth Dayana Mendoza-Matajira

2003 y 2012 escritos en español. se tuvo en cuenta la literatura disponible que aporta el conocimiento sobre los costos que se y comunitarios, instituciones y cuidadores profesionales.
Cúcuta-Colombia

Vol. 19

No. 2

Julio-Diciembre 2014 ISSN 0122-820X

PP: $51-58$

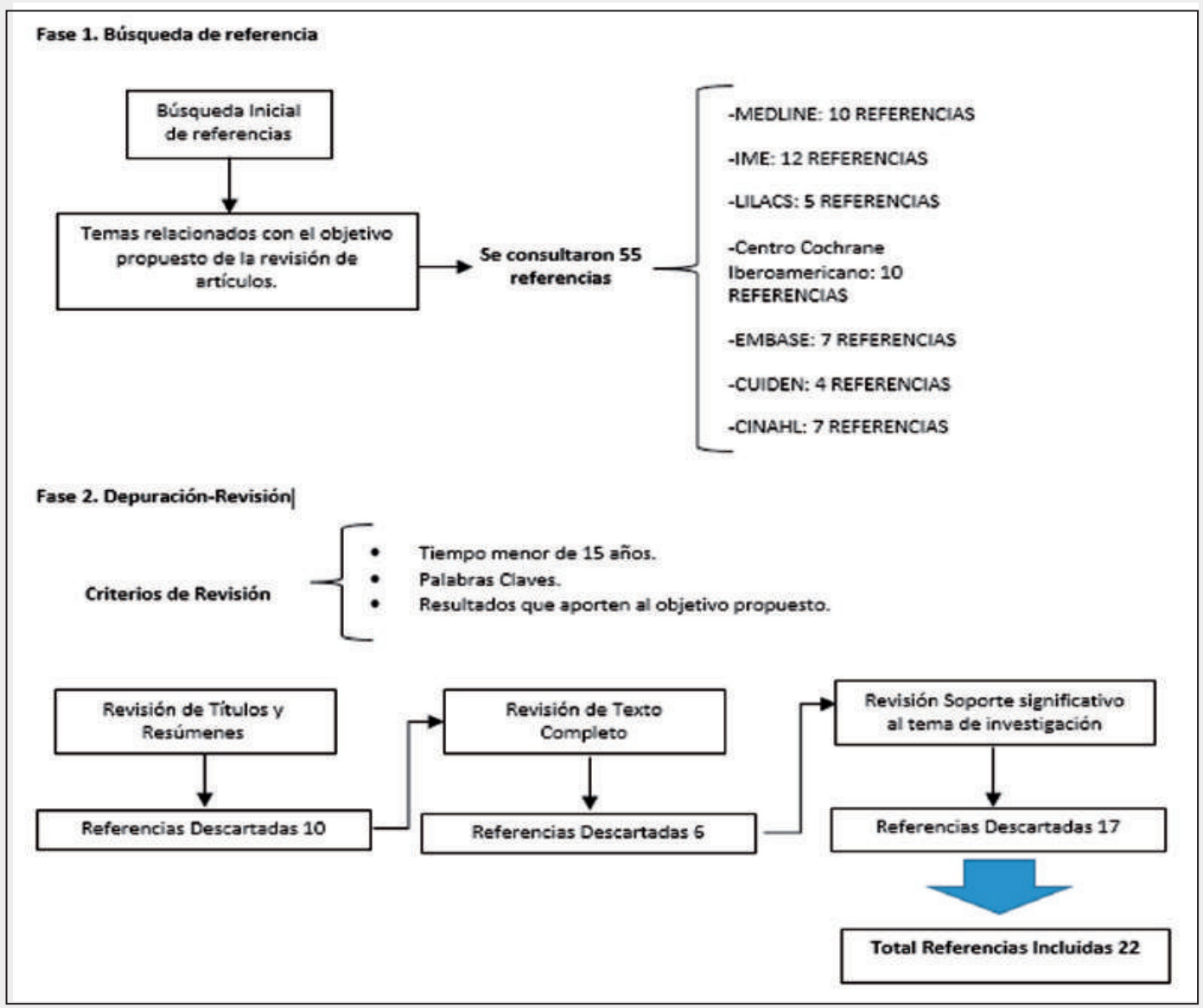

Figura 1. Diagrama de flujo de la información a través de las diferentes fases de una revisión sistemática Fuente: Autores

Se incluyeron 22 estudios con los siguientes criterios de inclusión: artículos menores de 15 años de publicación, palabras claves de acuerdo a la temática, resultados que aportaran al objetivo propuesto, revisión de títulos y resumen relacionado con el objetivo de la revisión del artículo.

A continuación se muestra la Tabla I de los artículos citados.

Tabla I. Artículos y datos más relevantes en la etapa de revisión

\begin{tabular}{|c|l|l|}
\hline Año & \multicolumn{1}{|c|}{ Revista } & \multicolumn{1}{c|}{ Título del Articulo } \\
\hline 2003 & $\begin{array}{l}\text { Revista de la sociedad } \\
\text { americana de geriatría. }\end{array}$ & $\begin{array}{l}\text { La pérdida de la independencia en las actividades de la vida } \\
\text { diaria en adultos mayores hospitalizados con enfermedades } \\
\text { médicas: aumento de la vulnerabilidad con la edad }\end{array}$ \\
\hline 2005 & Revista Aquichan. & $\begin{array}{l}\text { Reflexiones sobre el cuidado a partir del programa } \\
\text { "cuidando cuidadores" }\end{array}$ \\
\hline 2008 & Revista Salud Uninorte. & $\begin{array}{l}\text { Características de los cuidadores de personas en situación } \\
\text { de cronicidad. Cartagena (Colombia). }\end{array}$ \\
\hline 2010 & $\begin{array}{l}\text { Observatorios de Salud } \\
\text { Pública de Santander }\end{array}$ & Indicadores básicos de Salud \\
\hline 2011 & Revista Univ. Méd & $\begin{array}{l}\text { Proyección de costos en Colombia de la atención inicial del } \\
\text { síndrome coronario agudo. }\end{array}$ \\
\hline 2011 & MEDISAN & $\begin{array}{l}\text { Modelos de atención a pacientes con enfermedades } \\
\text { crónicas no transmisibles en Cuba y el mundo. }\end{array}$ \\
\hline
\end{tabular}


No. 2

Julio-Diciembre 2014 ISSN 0122-820X

\section{Resultados y análisis}

\subsection{Perspectiva del costo y el cuidado}

Una de las problemáticas que aumenta los costos es el declive del autocuidado de la persona hospitalizada, y el cambio que existe entre el ingreso y el alta. Según Covinsnky y otros (2003), en su estudio "Perdida de la independencia en las actividades de la vida diaria en adultos mayores hospitalizados", concluye que muchas personas de edad avanzada hospitalizadas, son dadas de alta con dichas funciones de la actividad diaria disminuida, los que tienen edad avanzada son menos propensos a recuperar estas funciones. Se diría que esto es un problema que aumenta los costos en la recuperación ambulatoria del individuo ya que tardaría más tiempo en tener una cotidianidad en sus labores y requerirá de un cuidador $y / o$ de terapias que antes de la hospitalización no necesitaba.

Por tanto la atención en enfermedades crónicas no trasmisibles abarcan muchos temas para llegar a una excelencia y disminución de costo de la vida de un individuo, según Pinto y otros (2005), en las reflexiones sobre el cuidado a partir del programa "Cuidando a los Cuidadores" refiere que es importante la labor de enfermería pero que aún mas es contar con el familiar en el que se desarrolle herramientas para mejorar su habilidad en términos de incrementar la capacidad de afrontamiento de manera tal que satisfaga las necesidades de la persona enferma, responda a la problemática familiar, mantenga su propia salud y fomente la buena relación cuidador-persona cuidada, reconociendo y estableciendo apoyos formales e informales, manejando sentimientos de inadecuación o culpa, y planeando su propio futuro y el de su grupo familiar.

La Organización Panamericana de la Salud (2013)menciona que las cifras van en aumento sobre las enfermedades crónicas no transmisibles, y que de las personas adultas el 90\% pueden requerir alguna atención relacionada con las enfermedades crónicas, así mismo alude sobre la mala atención de las mismas, y la falta de un diagnostico precoz lo cual repercute económicamente debido a las complicaciones, la dependencia, los reingresos que suelen tener los pacientes, así como la falta de adherencia al tratamiento.

Joval y otros (2011) menciona que en los modelos de atención a pacientes con enfermedades crónicas, se deben tener presente diversas estrategias que ayuden al paciente y la familia de tal forma disminuir los gastos que se generan por la atención de las enfermedades crónicas.

Es así como las ECNT se convierten en un gran reto, en donde se debe realizar programas educativos con el propósito de prevenir aquellas enfermedades, generando hábitos de vida saludables, orientándose a los determinantes de la salud de tal forma que los costos sociales no sean la problemática principal, según lo mencionado por Caballero, C., \& Alonso, L. (2010). Es por ello que el sector de la salud debe trabajar como prioridad la prevención de nuevos casos y la disminución de las complicaciones en los pacientes diagnosticados, el tratamiento eficaz de tal forma que se puedan obtener beneficios a corto, mediano y largo plazo, sugiriendo que cada país diseñe sus propias medidas y programas de prevención las cuales deben tener cobertura universal, Cordoba, A., y otros. (2008).

Por otro lado Moreno (2013), dimensiona que la prevención y disminución de las enfermedades crónicas no solo están a cargo del sector sanitario, si no que a su vez participan otros sectores sociales, políticos, públicos y privados ya que debido al incremento se convierte en un problema de salud pública del cual si no se toman medidas preventivas terminara siendo de alto costo para los países bajos que llevara consigo la pobreza extrema.

Además, la Organización Panamericana de la Salud (OPS) ha planteado la importancia de incluir a los gobiernos, a la sociedad civil, a la academia, a los organismos internacionales $\mathrm{y}$ al sector privado para combatir estas enfermedades y adelantar acciones que involucren infraestructura, educación, 
promoción de buenas prácticas y participación social. En tal sentido, la OPS convocó en 2011 al Foro Panamericano de acción sobre las enfermedades no transmisibles para promover iniciativas innovadoras, y para extender las prácticas más exitosas sobre la prevención y el control de estas enfermedades.

Los factores de riesgo asociados a las enfermedades crónicas no transmisibles, al igual que las acciones para su prevención, son asuntos que no se asumen únicamente por la persona directamente afectada, si no que trascienden y comprometen a las demás personas con las cuales comparte el paciente o enfermo potencial, e incluso a la sociedad en general; es ahí donde aparecen las llamadas externalidades (costos o beneficios externos), que justifican la intervención del Estado para prevenir y controlar factores de riesgo y de esta manera promover la salud y mejorar la calidad de vida. La identificación de estos costos y beneficios sociales son cruciales para definir el ámbito de las políticas públicas.

Por su parte, la OPS (2010), ha revelado que en las Américas se presenta una de las tasas más elevadas de mortalidad por ECNT, alcanzando un $44 \%$ del total de defunciones de hombres y mujeres menores de 70 años.

Colombia se encuentra en una situación preocupante en cuanto a las ECNT y sus factores de riesgo. Según el Boletín de Enfermedades Crónicas no Transmisibles, del Ministerio de Salud y Protección Social (2011), la enfermedad con la mayor mortalidad prematura es la enfermedad isquémica del corazón, con una tasa de mortalidad en menores de 70 años de 67,5 por cada 100.000 habitantes; esta está seguida por la enfermedad cerebrovascular, las enfermedades respiratorias crónicas y la diabetes mellitus, cuyas tasas de mortalidad fueron $31,1,26,9$ y 17,3 , respectivamente.

En el mismo informe del Ministerio de salud y protección social (2011) se presentan indicadores de prevalencia de sobrepeso y obesidad y para niños de 5 a 17 años, e indicadores de prevalencia de hipertensión en adultos. Entre otros factores de riesgo se destaca que el consumo de frutas y hortalizas (tres o más porciones de frutas y verduras por día) solo un $11,8 \%$ de la población nacional efectivamente lo hace. Por su parte, el 17,1\% de la población son fumadores activos, y el $12,2 \%$ de los adultos son consumidores de alcohol.

Según Roses (2012) las consecuencias de cualquier enfermedad recaen en primera instancia sobre el individuo que la padece y sobre su familia. El impacto económico se ve reflejado en la disminución del ingreso neto, lo que puede incluso comprometer el patrimonio familiar, pues por un lado, según la duración y la severidad de la enfermedad puede producirse una pérdida considerable de ingresos al no poder trabajar o al verse afectado el desempeño individual en el futuro; por otro lado, el tratamiento de la enfermedad acarrea para la familia costos de atención médica y de transporte, entre otros.

Para Suhrcke y otros (2006) mencionan en cuanto a la capacidad de mantener el gasto de consumo, la cuestión es si la familia posee algún mecanismo de seguro para afrontar los gastos asociados a la enfermedad sin ocasionar efectosadversossobre su consumopermanente. En el caso más dramático, los gastos médicos pueden significar un "gasto catastrófico" o "gasto médico empobrecedor", pues por el monto de recursos necesarios para la atención médica se pone en riesgo la capacidad de mantener el estándar de vida acostumbrado; la familia ve sacrificada una parte importante de su ingreso, sin que sea posible cubrir otras necesidades, y se ve obligada a endeudarse o a reducir su patrimonio mediante el gasto de su ahorro o la venta de activos.

\subsection{Conceptualización de la evaluación económica} ISSN 0122-820X PP: $51-58$

Arjona (2013) menciona la evaluación económica, definida como un conjunto de procedimientos de análisis dirigidos a evaluar el impacto de opciones alternativas sobre el bienestar de la sociedad y que se centra en la determinación de la eficiencia, es decir, 
No. 2 Julio-Diciembre 2014 ISSN 0122-820X

PP: 51-58 en la relación entre beneficios obtenidos en términos de salud y los recursos gastados, teniendo en cuenta que entre menos recursos se utilicen para lograr un objetivo, será mejor pues con los otros recursos se podrían plantear más objetivos. Al igual define términos como Costo-efectividad, el cual es el grado en que una determinada intervención o servicio sanitario logra lo que se quiere conseguir, además es el costo de un producto para disminuir algo, por ejemplo el costo de un producto para reducir el colesterol.

Datos del Instituto Nacional de la Salud de España (INSALUD), (1997), revela que el 46.9\% de las altas hospitalarias corresponde a personas mayores de 65 años, de las cuales la primera causa de ingreso son las enfermedades cardiovasculares. Por lo que el costo medio de las camas se eleva según el número de camas ocupadas por número de patologías crónicas.

El síndrome coronario agudo es una de las ECNT que son de alto costo para el país, Castellanos (2011), menciona que en nuestra sociedad los resultados arrojados del costo global promedio general es de 7.781.230 pesos, entre los costos médicos y los medicamentos para ello este artículo propuso llevar información de lo que gasta Colombia, con el fin de que las instituciones usen las tecnologías para detección y manejo de estas patologías creando protocolos y guías que permitan mejorar la atención clínica al paciente y protección adecuada de los recursos del Sistema General de Seguridad Social en Salud. Otro de los estudios encontrados en Colombia fue "características de los cuidadores de personas en situación de cronicidad en Cartagena”. Montalvo (2008), quien comenta que el incremento de las enfermedades crónicas no trasmisibles conlleva un mayor costo y aumenta la carga de aquellas personas que asumen el cuidado del enfermo, la mayoría de los cuidadores son mujeres que a la vez son madre-esposa-hija, ama de casa y/o trabajadora, con la dificultad para compatibilizar las distintas responsabilidades todo esto repercute en la vida de ellas tanto personal, social y económicamente. Por todo lo anterior los costos en Colombia va en aumento, por la falta de programas o guías bien aplicadas o el conocimiento de ellas para emplearlas y poder disminuir los costos en la carga de las enfermedades crónicas no trasmisibles, evidenciándose en el alto consumo de fármacos de terapia cardiovascular, la ocupación hospitalaria, la falta de adherencia, el manejo hospitalario de patologías mentales y la detección tardía de enfermedades y complicaciones, además el contraste de costos para los pacientes y cuidadores.

Se considera más beneficioso para la institución atenderlo en casa; aunque para la familia aumenta el costo por desplazamiento a los controles médicos, los cuidados adicionales que se deben ofrecer en el hogar y los insumos requeridos para el cuidado.

Según lo mencionado en el Observatorio de la Seguridad Social (2012), las consecuencias de cualquier enfermedad recaen en primera instancia sobre el individuo que la padece y sobre su familia. El impacto económico se ve reflejado en la disminución del ingreso neto, lo que puede incluso comprometer el patrimonio familiar, pues por un lado, según la duración y la severidad de la enfermedad puede producirse una pérdida considerable de ingresos al no poder trabajar o al verse afectado el desempeño individual en el futuro; por otro lado, el tratamiento de la enfermedad acarrea para la familia costos de atención médica y de transporte, entre otros.

\section{Conclusiones}

Sin duda alguna las enfermedades crónicas no transmisibles generan gran parte de los costos sanitarios, el costo aumenta para cada paciente dependiendo de la clasificación económica, así mismo se connota a nivel nacional el aumento de las enfermedades crónicas no transmisibles y las consecuencias que traen consigo en el sector de la salud tanto en la atención como en el costo financiero.

El pronóstico es el aumento de personas afectadas por estas enfermedades debido a la mayor exposición a factores de riesgo 
modificables como la hipertensión arterial, la diabetes, el sedentarismo, la obesidad, niveles elevados de colesterol, tabaquismo, alcoholismo y estrés.

Por esta razón es importante que el Sistema de Salud valore la gravedad de la situación y ya sea por razones humanas o económicas implemente políticas, planes y programas para prevenir la aparición de las ECNT en la población así como de cuidado efectivo para la atención de pacientes que ya han sido afectados por estas enfermedades.

Teniendo en cuenta que hay evidencia que están en juego las acciones de auto cuidado durante la hospitalización, es necesario que el papel de enfermería este encaminado para cubrir las necesidades en las cuales el paciente tiene falencia y potencializar las que el mismo pueda cubrirse, esto con el fin de que haya menos secuelas de la hospitalización como la perdida de las funciones o actividades de la vida diaria.

\section{Referencias}

Arjona, C. (2013). Repercusiones económicas de las enfermedades crónicas. El médico Interactivo. Recuperado de: http://www. elmedicointeractivo.com/ap1/emiold/ informes/gestion/ repercusiones.htm.

Castellanos, C. (2011). Proyección de costos en Colombia de la atención inicial del síndrome coronario agudo. Revista Univ. Méd, 52 (1): 36-43.

Caballero, C.V., \& Alonso, L.M. (2013). The Millennium Development Goals and Health Crisis. Revista Salud Uninorte, 29(1), 6-8. Recuperado de: http://www.scielo. org.co /scielo.php?script=sci_arttext\& pid $=\mathrm{S} 0120-55522013000 \overline{1} 00001$ \&lng $=\mathrm{es} \&$ tlng $=\mathrm{en}$.

Córdova, J. Á., Barriguete, J. A., Lara, A., Barquera, S., Rosas, M., Hernández, M., \& Aguilar, C. A. (2008). Las enfermedades crónicas no transmisibles en México: sinopsis epidemiológica y prevención integral. Salud pública de México, 50(5), 419-427.
Covinsky, K., Palmer R., Fortinsky, R., Counsell, S., Stewart, A., Kresevic, D., et al. (2003). La pérdida de la independencia en las actividades de la vida diaria en adultos mayores hospitalizados con enfermedades médicas: aumento de la vulnerabilidad con la edad. Revista de la sociedad americana de geriatría, 51(4), 451-8.

Jova, R., Rodríguez, A., Díaz, A., Balcindes, S., Sosa, I., Pol, V., Van der Stuyft, P. (2011). Modelos de atención a pacientes con enfermedades crónicas no transmisibles en Cuba y el mundo. Revista MEDISAN, 15(11), 1610.

Joval, R., Rodriguez, A., Diaz, A., Balcindes, S., Sosa, I., De Vos, P., \& Van de Styft, P. (2011). Modelos de atención a pacientes con enfermedades crónicas no transmisibles en Cuba y el mundo. Revista MEDISAN, 1609. 1620.

Ministerio de Salud y Protección Social. (2011). Primer Boletín de Enfermedades Crónicas No Transmisibles. Recuperado de: http://www.gestarsalud.com/cms/files/ PRIMER\%20 BOLET\%C3\%8DN\%20ECNT. pdf.

Ministerio de Salud y Protección Social. (2012). Guía de Atención Integral basada en la Evidencia, para la Detección temprana. Recuperado de: http://gpc.minsalud.gov.co/ guias/ Documents/Distrofia\%20Muscular/ GPCDM\%2037\%20para\%20usuarios.pdf.

Montalvo, A., Flórez, I. (2008). Características de los cuidadores de personas en situación de cronicidad. Cartagena (Colombia). Revista Salud Uninorte. 24(2), 181-190.

Moreno, J. M. (2013). Real Academia de Medicina de la Comunidad Valenciana. La pandemia de enfermedades crónicas no transmisibles. Retos de salud pública y nuevos paradigmas en el siglo XXI. Recuperado de: http://www.ramcv.com/Discursos/Martin $\% 20$ Moreno.pdf.

Observatorio de salud pública de Santander. (2010). Recuperado de: http://www.ob-
Julio-Diciembre 2014 ISSN 0122-820X PP: 51-58 
No. 2

Julio-Diciembre 2014 ISSN 0122-820X

PP: $51-58$ servatorio.saludsantander.gov.co/index.php/ noticias /osps/158-la-secretaria-de-salud-desantander-y-el-observatorio-de-salud-publica-de-santander-socializaron-los-indicadores-basicos-de-salud-2010.

OMS/OPS/CEPAL. (2011). Taller: Economía de la prevención de enfermedades no transmisibles y sus factores de riesgo. Recuperado de: http://www.paho.org/hq/ index.php?option $=$ com_content \&view $=$ ar ticle \&id=6232: tallereconomia-prevencionenfermedades-no-transmisibles-sus-factoresriesgo-2011\&Itemid $=43$ 39\&lang $=$ es.

Organización Mundial de la Salud -OMS. (2008). Prevención y control de las enfermedades no transmisibles: aplicación de la estrategia mundial. Recuperado de: http:// apps.who.int/iris/bitstream/10665/26218/1/ A61_8-sp.pdf.

Organización Mundial de la Salud -OMS. (2011). Enfermedades no transmisibles. Recuperado de: http://www.asivamosensalud. org / media/s a n tafe / p ublic a cion / f834bc7de5778255b2b571631083feaf.pdf.

Organización Panamericana de la Salud -OPS. (2010). Grupo de Trabajo: Enfermedades No Transmisibles Mercosur y Países Asociados. Recuperado de: http://epi.minsal.cl/epi/ Onotransmisibles/vent/VENT_Mercosur_ FINAL_17092011.pdf.

Organización Panamericana de la Salud. (2013). Cuidados innovadores para las condiciones crónicas: Organización y prestación de atención de alta calidad a las enfermedades crónicas no transmisibles en las Américas. Recuperado de: http:// www.paho.org/hq/index.php?option $=\mathrm{com}$ docman\&task $=$ doc_view\&gid $=22257+\& \overline{I t}$ emid $=999999 \&$ lang $=$ es.

Pinto, N., Barrera L., Sánchez B. (2005). Reflexiones sobre el cuidado a partir del programa "cuidando cuidadores". Revista aquichan. 5(1), 128-13.

Suhrcke, M., Nugent, R. A., Stuckler, D., $\&$ Rocco, L. (2006). Chronic disease: an economic perspective. Recuperado de: M Suhrcke, RA Nugent, D Stuckler, L Rocco - 2006 - citeulike.org.

Observatorio de la Seguridad Social. (2012). Economía de las enfermedades crónicas no transmisibles Implicaciones para Colombia. Medellín AÑO 11 № 24. ISSN 1657 - 5415. Recuperado de: http://www.udea.edu.co/ portal/page/portal/BibliotecaInvestigacion/ CienciasEconomicas/GES/Documentos1/ Observatorio_24.pdf. 